\title{
KEY TECHNOLOGIES OF MECHANICAL FAULT DIAGNOSIS SYSTEM BASED ON CLOUD COMPUTING
}

\author{
Yan $\mathrm{Li}^{1}$, Lei Wang ${ }^{2}$, Wei $\mathrm{Li}^{3}$ \\ 1,2,3 Hebei Institute Of Communications, Hebei 051430, China \\ Email: liyan hbic@126.com
}

\begin{abstract}
In order to solve a series of adverse consequences caused by mechanical failure in practical engineering, intelligent and convenient use of various data to monitor and analyse the causes of mechanical failure, a mechanical fault diagnosis and analysis system based on cloud computing is designed by taking the typical rotor system dynamics model as the research object. Firstly, the relevant data are consulted and the related theories and research methods of cloud computing and mechanical fault are learned. Then, the Hadoop platform is built for the rotor system dynamics model and MapReduce programming is carried out. Combining the cluster characteristics of cloud computing, FFT (Fast Fourier Transform) algorithm is studied. Finally, the computing performance of Hadoop platform is tested and analysed to verify the correctness of FFT parallelization and parallel computing performance. The research results show that the Hadoop platform of mechanical fault diagnosis system has the characteristics of high real-time performance and high reliability, and shows the advantages of parallel FFT algorithm based on MapReduce compared with other solutions. The innovation is that the whole system adopts advanced cloud computing concept and chooses the best MapReduce-based Hadoop platform. The research results also confirm the excellent performance of this system.
\end{abstract}

Keywords: Mechanical Fault Diagnosis; Cloud Computing; Hadoop Framework; Mapreduce Programming.

\section{Introduction}

Large-scale rotating machineries, such as steam turbine, blower, generator and compressor units, are large and complex mechanical equipment. They are also key machineries in the pillar industries of national economy, such as petroleum, machinery, metallurgy, transportation, chemical industry, power generation etc. Because many factors are involved in the manufacture, installation, operation and maintenance of machinery, it is unavoidable that faults will occur. Once a fault occurs, it will cause a series of chain reactions. It will cause production process interruption and shutdown, and even lead to serious catastrophic accidents, resulting in huge economic losses and adverse social impact.

Therefore, it is more and more important to monitor the condition of machinery and forecast the faults in time in the modern management of enterprises [1].

In recent years, great progress has been made in the study of mechanical failure. Since the 1960s when the United States began to carry out study on mechanical failure, mechanical fault diagnosis technology has formed a new discipline - machine fault diagnostics. The main task of this subject is to study how to distinguish the technical status of mechanical devices [2] under the condition of limited information from the basic theory, physical mechanism, mathematical methods and technical means.

Cloud computing is a completely new network service mode, which has been widely used in medical, transportation, education and other industries, and has achieved good results. For most enterprises, using resources through cloud computing can reduce the overhead cost of resources and shorten the cycle of enterprise system information construction. For remote diagnosis system, cloud computing technology can make the system build on demand to obtain resources and expand in a flexible way; and remote diagnosis system can also give full play to the mass storage, supercomputing, dynamic expansion, high reliability and availability of cloud computing. Through cloud computing technology, all kinds of resources needed by fault diagnosis system can be aggregated, and a huge data processing and storage center can be built, which can realize data supercomputing and mass storage. Generally speaking, cloud computing technology makes enterprises no longer need to buy expensive hardware equipment, but only need to rent equipment from cloud service providers to build their own fault diagnosis platform 
conveniently and quickly, which reduces the investment cost of facilities, and achieves the maximization of enterprise benefits. Moreover, cloud computing technology can share information among enterprises, and even achieve collaborative work among different enterprises, effectively eliminating the "island" phenomenon in industry information systems [3].

In recent years, some large domestic enterprises have begun to gradually build their own monitoring centers for machinery and equipment, and make real-time monitoring and control of machinery, and machinery information management level is constantly improving. For most small and mediumsized enterprises, due to their limited production scale and financial resources, it is impossible to build or develop professional mechanical monitoring and fault diagnosis service centers or expert diagnosis systems. The emergence of cloud computing technology provides solutions and technical support for this problem. Originally, for individuals or businesses, in order to increase computing and storage capacity, they need to buy more computers or servers. With the development of cloud computing technology, more and more enterprises or individuals no longer need to upgrade hardware facilities and software, but only need cloud computing service providers to complete, thus greatly reducing operating costs. At present, cloud computing technology has been widely used in manufacturing, medical, transportation, finance, education and other industries. With the advent of the information age, mechanical condition monitoring and fault diagnosis based on network technology has become a trend of development. The application and research of mechanical condition monitoring and fault diagnosis based on cloud computing technology has far-reaching significance for both modern industrial production and the development of cloud computing [4].

The basic theory and development status of cloud computing technology, as well as the Hadoop framework and MapReduce parallel programming model of cloud computing platform are mainly analysed, and the implementation method of mechanical on-line monitoring and fault diagnosis system in cloud computing environment is discussed and analysed. It mainly includes the following research contents: the basic principle and development status of cloud computing, the key technology of Hadoop framework, and the implementation strategy of cloud computing development environment; the construction of cloud computing environment uses the framework. Hadoop 0.20 cloud computing network topology consists of four PCs, one as Name Node node and three as Data Node node; the advantages of cloud computing technology in mechanical fault diagnosis and implementation strategies are analyses, and implementation methods of parallel FFT algorithm in the Map Reduce programming model of the cloud computing platform Hadoop are discussed; the application program of mechanical fault diagnosis is developed under the Hadoop framework of cloud computing platform, and the program is deployed and tested.

\section{Mechanical Fault and Diagnosis Technology}

Fault diagnosis technology is a modern engineering discipline which integrates many disciplines such as mathematics, mechanics, measurement and control technology, signal processing technology etc. It mainly makes comprehensive processing and analysis through the operational information such as vibration, noise, temperature and working parameters, to determine the working state, fault type, fault severity and solution. In the 1980s and 1990s, with the continuous development and rapid popularization of computer technology, computercentered online mechanical monitoring and diagnosis technology emerged. Relying on network technology and adopting fault diagnosis technology and signal processing method, the mode of fault diagnosis has changed essentially. There are three stages: single monitoring and diagnosis mode, distributed monitoring and diagnosis mode, and remote monitoring and diagnosis mode.

\subsection{Dynamic model of rotor system}

There are many kinds of rotating machinery, such as generator, gas and steam turbine, steam turbine, diesel engine, ventilator etc. Most of the faults are caused by rotor and bearing faults. The main types of faults include rotor unbalance, rotor misalignment, rotor eccentricity, feed chain faults, spindle components faults, etc. For relatively simple rotating machinery such as rigid rotor, the mechanical model of the rotor can be simplified to a single disk rotor dynamic model: the disk is mounted on a mass-free elastic rotating shaft, and the two ends of the rotating shaft are supported by rigid bearings and bases. This model is taken as an example here.

The motion of the rotor is a combination of two kinds of motion: one is the rotation of the rotor itself around the horizontal axis, and the other is the bow rotation of the plane winding composed of the bending axis $\mathrm{AOB}$ and the bearing connection AOB. Set the mass of the disk to be $\mathrm{m}$, the elastic restoring force $F$ is:

$$
F=-\mathrm{kr}
$$

In the above formula, $\mathrm{k}$ refers to stiffness coefficient of rotor; $r$ indicates centroid distance of rotor.

The differential equation of circular motion of a single disk rotor system can be expressed as: 


$$
\left\{\begin{array}{l}
m \ddot{x}=F_{x}=-k x \\
m \ddot{y}=F_{y}=-k y
\end{array}\right.
$$

Set $\omega_{n}^{2}=k / m$, and then there is:

$$
\left\{\begin{array}{l}
\ddot{x}+\omega_{n}^{2} x=0 \\
\ddot{y}+\omega_{n}^{2} y=0
\end{array}\right.
$$

Its solution can be written as:

$$
\left\{\begin{array}{l}
x=X \cos \left(\omega_{n} t+\theta_{x}\right) \\
y=Y \cos \left(\omega_{n} t+\theta_{y}\right)
\end{array}\right.
$$

$\mathrm{X}$ and $\mathrm{Y}$ represent the amplitude, and $\theta_{x}$ and $\theta_{y}$ are initial phase, and are related to the initial state of the rotor. Formula (4) shows that the center of a single disk rotor oscillates at the same frequency in two perpendicular directions, and the trajectory of the center of the rotor is ellipse, which is a natural vibration called precession (whirl).

\subsection{Common mechanical faults and their characteristics}

Mechanical fault diagnosis is an important research field of mechanical engineering, which is used to find the causes of faults on machines. It is especially suitable for the most common types of faults in rotating machinery. To find out the most possible cause of failure, many methods are used for data acquisition, including vibration monitoring, thermal imaging, oil particle analysis, etc. These data are usually processed by spectrum analysis, wavelet analysis, and time domain waveform analysis, because spectrum analysis only focuses on the spectrum distribution and does not need phase information. The results of the analysis are used for failure analysis to determine the initial cause of the failure, such as diagnosing a bearing fault, it is likely that the bearing itself is not damaged during installation, but another installation error, such as eccentricity, causes the bearing damage. Diagnosing the damage condition of the bearing is not enough for the goal of precision maintenance machinery.

The fundamental cause of the failure needs to be identified and remedied. If the fault origin is not known, as a result, even if the bearing is replaced, the same failure will occur soon, and the machine will suffer greater damage and potential danger. Of course, in the data acquisition stage, the cause of failure is also clearly visible through spectrum analysis, but the actual situation is not always the case. Mechanical failure refers to an abnormal state in which the machine cannot work normally according to the expected performance indicators in the use process.

There are many reasons for failure, such as design, process, quality, or some defects in application, etc., which are all potential factors.
At the same time, the users and operators of the machine will also lead to machine failure, and human factors must also be considered. The bathtub curve of mechanical failure rate is shown in Figure 1 as follows:

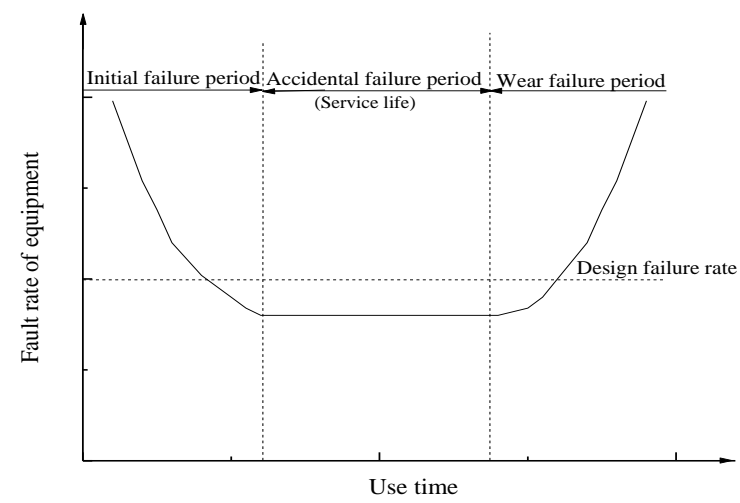

Figure 1: Bath tub graph of mechanical failure rate

The probability of failure of the mechanical equipment in use can be roughly divided into three stages: initial failure stage, accidental failure stage, and wear failure stage. Partial failure modes are caused by component failures, and a component usually has several failure modes. This failure mechanism can be caused by many different types, usually by multiple factors, such as corrosion, abnormal contact current during welding, fatigue fracture, mis-operation, dust accumulation, blockage and so on. In theory, the real failure can be traced back to some human factors in most cases, such as unreasonable design, mis-operation, poor management, unreasonable maintenance, etc. [5].

Some types of mechanical failure mechanisms include bending, excessive burning, fracture, creep, loosening, thermal shock, corrosion, corrosion cracking, and various types of fatigue. Each of them can produce different types of fracture. The loading mode and design factors of machinery are very important, because stress concentration can magnify the load to a very high level. With the increase of time, more failure causes can be known. An abstract model for describing failure causes and symptoms development is established. The more complex the working condition of machinery, the more necessary it is to understand its failure causes to ensure its normal operation. Common mechanical equipment faults are: rotor misalignment, rotor imbalance, oil whirl, oil film oscillation, surge, etc. [6].

\subsection{Vibration signal analysis method}

In the operation of machinery, some physical properties such as vibration, noise, temperature and pressure will change regularly with time. Effective analysis of these useful feature information and establishment of a certain relationship model are the basis of mechanical fault diagnosis. Vibration analysis is usually used in traditional fault diagnosis, 
because vibration is one of the important performance indicators of mechanical equipment. Vibration strength has a great relationship with fault. Vibration monitoring is easy to realize in technology and relatively mature in theory. Vibration signal is a kind of dynamic data. Vibration amplitude contains abundant information, so it has universal applicability for signal analysis and processing [7].

Compared with the methods of oil monitoring, temperature monitoring and strain monitoring, vibration monitoring has the characteristics of realtime, high reliability and wide application in current methods of mechanical fault diagnosis. The advantage of this method is that it can detect the abnormal vibration phenomena of components in different parts without special signal source, realtime measurement, signal testing and processing are simple and feasible. The main methods of vibration monitoring are time domain analysis, frequency domain analysis and time-frequency domain analysis according to type. The following is a brief introduction to these data analysis methods.

The most basic and direct expression of time domain analysis signal is to describe the change of physical quantity with time as independent variable, while time domain analysis is to decompose the signal in time domain to obtain intuitive and accurate results, which can be expressed as function analytic description or waveform diagram. Time domain diagrams describe the dynamic information of mathematical functions, physical signals, economic or environmental data varying with time. Time domain analysis has become the original basis of mechanical fault diagnosis because of its large amount of information, intuitiveness, easy to understand and other characteristics. Statistical eigen values of time-frequency analysis include mean square, mean, and variance of instantaneous waveform.

Frequency domain analysis is based on the analysis of phase spectrum and frequency spectrum of data from the frequency structure of signal. It is developed in the demand of signal encryption and medium transmission. When analysing a signal sequence with time domain analysis method, the signals are identical in time domain direction, but not necessarily in frequency domain direction. The information provided by time domain analysis method is limited. It can only roughly judge whether there is a fault or not, but cannot judge the location of the fault. Frequency domain analysis can find the relevant amplitude and phase information are obtained and the fault source by decomposing the time domain signal in abscissa coordinate with time into domain signal in abscissa coordinate with frequency. Frequency domain diagram is used to describe the frequency of signal in a given frequency band. Frequency domain analysis method is widely used in the fields of electronic engineering, control system engineering, and statistics.
Frequency domain analysis is more concise and profound. Dynamic signals are transformed from time domain to frequency domain mainly by Fourier series and Fourier transform. The aperiodic signal passes through Fourier transform and the periodic signal passes through Fourier series.

Time-frequency domain analysis of deterministic signals can be analysed in both time and frequency domains, but non-stationary signals hope to combine time-domain analysis with frequency-domain analysis, so that they can reflect both the frequency content of signals and the law of frequency content changing with time, and at the same time reflect the corresponding relationship between the signal in time and frequency domains. Time-frequency analysis method can clearly describe the relationship between signal frequency and time. The basic idea of time-frequency analysis is the combination of the design time and frequency described by the energy density or intensity signals at different times and frequencies. By using time-frequency distribution to analyse signals, instantaneous frequencies and amplitudes at each time can be given to complete the time-frequency signal research. STFT (Short-time Fourier transform), wavelet analysis and WHT (Walsh-Hadamard transformation) are commonly used time-frequency analysis algorithms in industry [8].

\section{Cloud Computing Theory and Model}

\subsection{Cloud computing theory}

Cloud computing is a network-based computing method, which can provide shared hardware and software resources and information services to users in an on-demand and scalable manner through the network. Cloud computing can use the Internet to decompose a huge program into countless smaller subroutines, which are then handed over to a system composed of multiple servers to feed back the processing results to users after search, calculation, and analysis. This technology enables network service providers to use ordinary computers to process huge amounts of data information in a very short time to achieve the same processing effect as supercomputers. In cloud computing, users' programs and data are no longer running or stored on personal computers, but hosted in the cloud. With personal computers or portable devices connected to the cloud via the Internet, users can access applications and information services from anywhere in the world. The core idea of cloud computing is to uniformly schedule and manage a large number of computing resources connected to the network, forming a huge computing resource pool to provide services for users. Cloud computing has the advantages of fast, convenience, independence, low cost, sharing, etc. [9]. 
Table. 1 Comparison between cloud computing and

\begin{tabular}{|c|c|c|}
\hline & Traditional way & $\begin{array}{l}\text { Cloud } \\
\text { computing }\end{array}$ \\
\hline $\begin{array}{l}\text { Realization } \\
\text { mode }\end{array}$ & $\begin{array}{l}\text { Purchasing } \\
\text { equipment and } \\
\text { developing } \\
\text { system }\end{array}$ & $\begin{array}{l}\text { Purchase of } \\
\text { external } \\
\text { services }\end{array}$ \\
\hline $\begin{array}{l}\text { Business } \\
\text { mode }\end{array}$ & $\begin{array}{l}\text { Payment of } \\
\text { equipment and } \\
\text { labour costs }\end{array}$ & $\begin{array}{l}\text { On-demand } \\
\text { service }\end{array}$ \\
\hline $\begin{array}{l}\text { Technology } \\
\text { mode }\end{array}$ & single user & $\begin{array}{l}\text { Multi-user, } \\
\text { flexible }\end{array}$ \\
\hline $\begin{array}{l}\text { Technologica } \\
\text { l capability }\end{array}$ & Specific scale & $\begin{array}{l}\text { Super-large } \\
\text { scale }\end{array}$ \\
\hline Extensibility & Non-expansible & $\begin{array}{l}\text { Extensible and } \\
\text { dynamically } \\
\text { scalable }\end{array}$ \\
\hline Running cost & High & Very cheap \\
\hline
\end{tabular}

The services provided by cloud computing are classified into three layers: SaaS (Software as a Service), PaaS (Platform as a Service) and IaaS (Infrastructure as a Service). IaaS provides cloud services to users through infrastructure hardware resources provided by cloud service providers. PaaS is to provide computing, development environment, and other platforms as services. Users can develop their own applications directly through the platform without purchasing servers, and provide them to other users. SaaS refers to the service mode that the service provider hosts the application software on other servers and then provides the software to customers through the Internet.

The software provided is only used when the user needs it. The user does not need to spend a lot of money to purchase hardware and software, but only pays a certain rental fee.

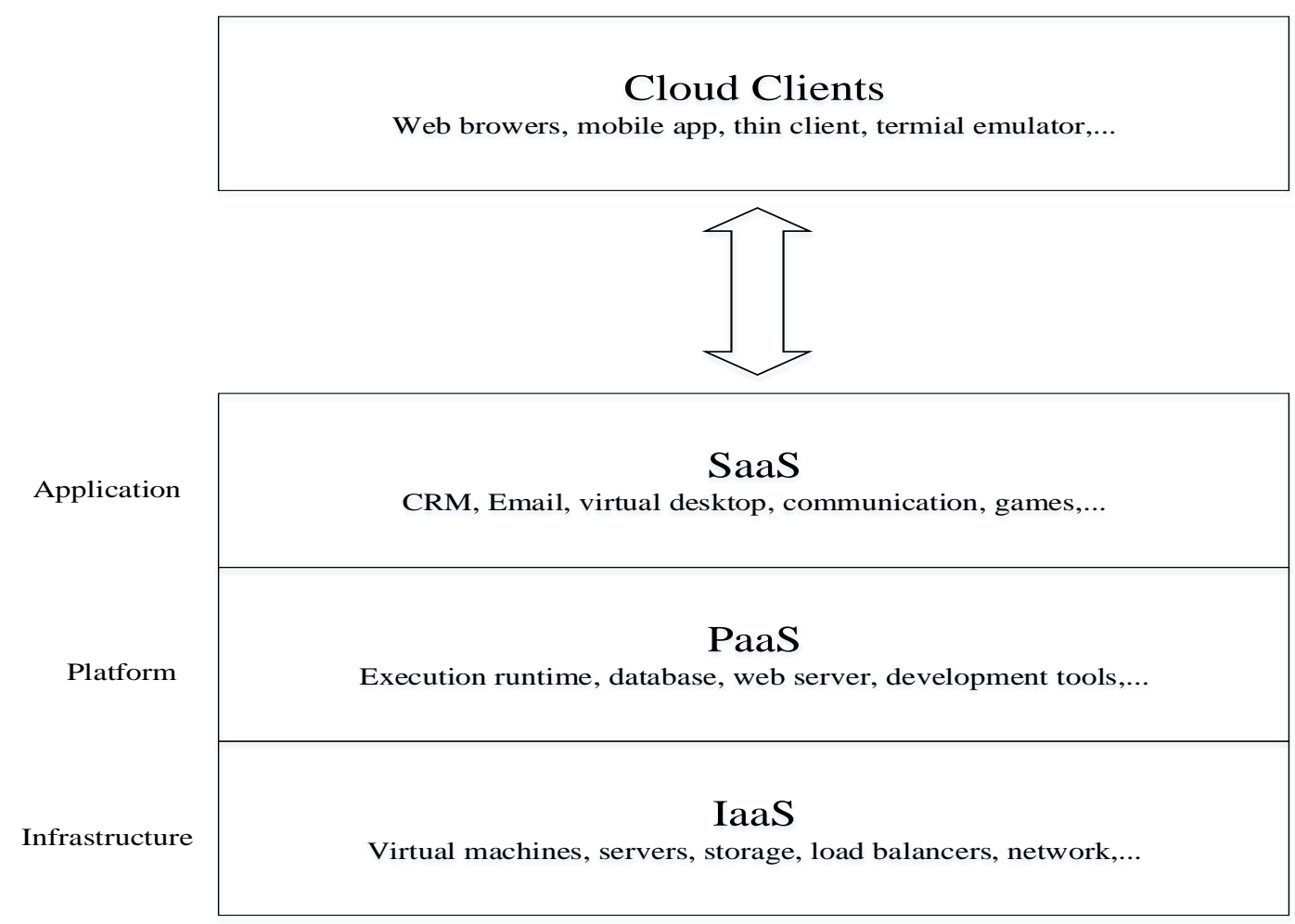

Figure 2: Cloud computing service hierarchy

The concept of cloud storage is extended and developed on the basis of the concept of cloud computing. In the development of cloud computing, the development of cloud storage can be seen everywhere. Eric Schmidt, CEO of Google, pointed out in his concept of cloud computing that "cloud computing distributes data on a large number of distributed computers, so that data storage has strong scalability". NIST (National Institute of Standards and Technology)'s definition of cloud computing also refers to "an application mode that connects resources (such as servers, storage, applications and services) through the network".

Thus, cloud storage is an important part of cloud computing.

Cloud storage is not only a storage technology or device, but also a service innovation.

The definition of cloud storage consists of two parts:

Firstly, in terms of user-oriented service form, it is an application mode providing on-demand services. 
Users can connect cloud storage resources through the network to realize the storage of user data in the cloud at anytime and anywhere.

Secondly, in the construction of cloud storage services, it achieves massive, flexible, low cost and low energy consumption shared storage resources through distributed, virtualized, intelligent configuration and other technologies.

By using cloud storage, enterprises can gain the following benefits: saving the cost of purchasing storage devices, shortening the system construction cycle, and reducing the cost of human and resources to maintain storage devices [10].

Cloud storage services have also developed rapidly, and formed a variety of open source and commercial cloud storage platforms.

Typical cloud storage platform interfaces usually include user data access control interface and user resource operation interface.

At present, the mainstream cloud storage services abroad include Amazon's S3 service, Google Storage service and Microsoft's Live Mesh service, while the domestic cloud storage services mainly include Aliyun, Baidu cloud, Jinshan Express and so on. Amazon's S3 supports REST and SOAP-type interfaces, including Get, Put, List, Delete and Head. Google Storage supports REST-type API interfaces, which can be operated using standard methods such as Delete, Get, Head and Put.

Microsoft's Live Mesh also provides a set of REST-style API interfaces, which also operate objects through GET, POST and other methods.

Domestic cloud storage services such as Baidu Cloud and Jinshan Express also provide developers with a complete set of REST API interfaces, through which data on the cloud disk can be manipulated in a variety of ways.

Files are a set of named information stored on a certain medium (such as disk, CD, etc.).

They are a form of existence that computers can recognize and process.

The software modules responsible for storing and managing these files in the operating system are called file systems.

File system is a system that allocates and organizes the storage space of files. It is mainly responsible for the storage of files and the retrieval and protection of stored files.

It is convenient for computer users and operating systems to manage and use files.

Common file systems are Linux file system, virtual file system, FUSE file system and so on.

\subsection{Key technologies of cloud computing}

The idea of Map Reduce is a parallel computing model first proposed and continuously promoted by Google.

Map Reduce computing process has three parts: Map process, Combine process and Reduce process, which is a mapping and specification process.

Map Reduce uses the idea of divide and conquer, dividing a business processing requirement into two stages: dividing a large task into several sub-tasks, namely Map stage; after parallel execution, merging the results, namely Reduce task, which is a summary of these map tasks [11].

As an operation, the set of parallel input files is split into several blocks. If there are so many single files, it will affect the search time, and then it will split into many blocks.

The process of partitioning does not know the internal logical structure of the input file and how to allocate it, such as splitting the linear text file at the boundaries of arbitrary bytes.

Each class automatically creates a new task, and when a separate task is completed independently, a corresponding task is opened to configure the operation. Using classes to read files at the same time generates key-value pairs of input and output from the given classes.

When the task operation node outputs key-value pairs, these records are displayed in memory. In order to improve the efficiency of parallel computing, sometimes it is necessary to make full use of a merged class to perform functions.

When a combiner is used, the key-value pairs of tasks are not immediately written to the output file. Instead, they are included in the list, with a list for each index key value.

When a certain number of key pairs are ready, the buffer is read and displayed by the method key of each key combiner, that is, the Combine process.

Reduce process means that when a task begins, its input file is divided into data blocks and run in a large number of files at each node. If it runs in a distributed model, first, it needs to copy to the local file system.

Once all the data are attached to a local file, the file is then categorized.

As a given key value, the key pair is continuous, which makes the actual operation very simple.

Files are read sequentially, and key values of input files are read through iterators and passed to different tasks until the next key value ends. 


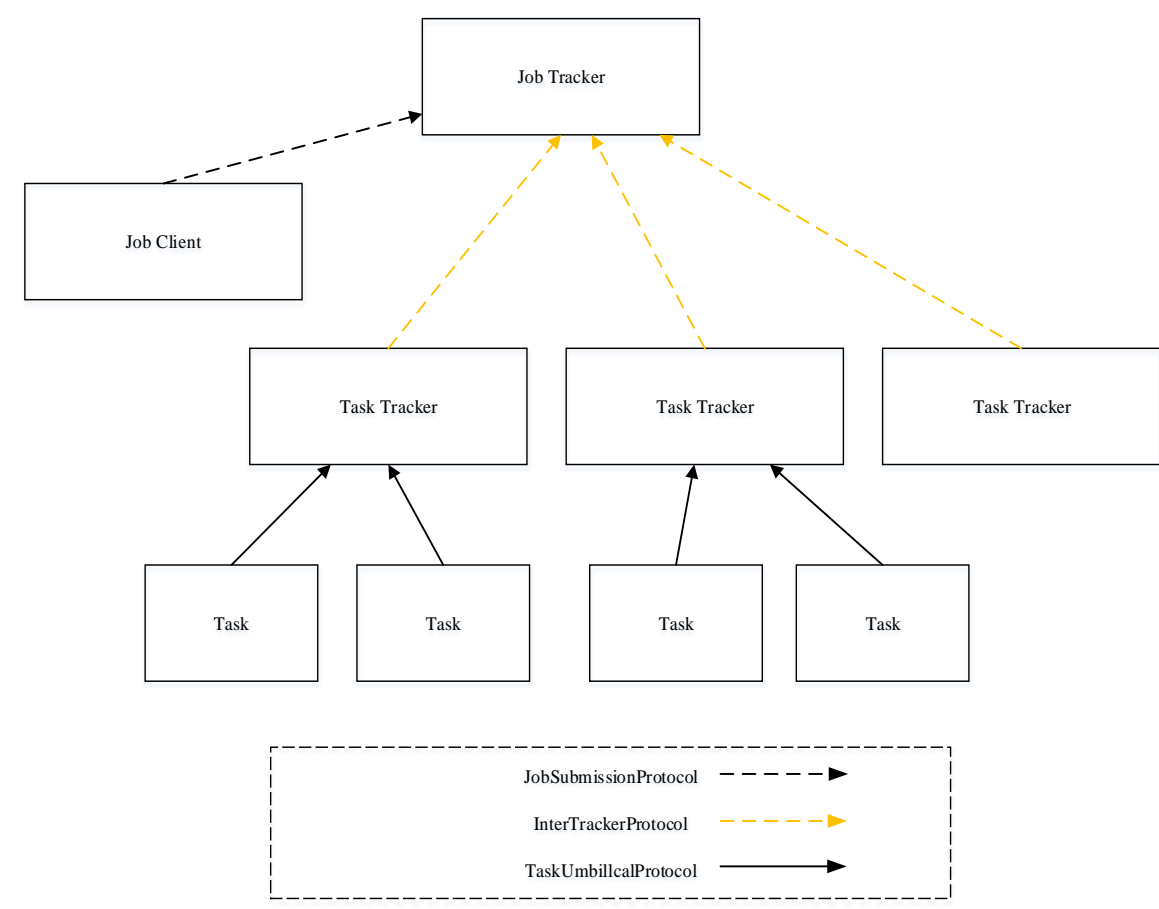

Figure 3: Map Reduce overall task control process

The emergence of MapReduce cloud computing model can solve the problems of task allocation, fault tolerance, and merging of computing results when processing big data sets. When it comes to the advantages of MapReduce, there are two main aspects: one is that MapReduce can be used to deal with large-scale data, hide complex details, and simplify development and programming; the other is that MapReduce has good scalability, and it can automatically carry out load balancing, data redundancy backup, and failed task restart process.

HDFS is a distributed storage support system for Hadoop applications.
An HDFS cluster consists of two parts: NameNode node for file system management and DataNodes node for data storage. HDFS framework mainly deals with the interaction between users and administrators and cluster management [12].

Hadoop is developed in Java language. HDFS, MapReduce and HBase in Hadoop are the open source implementations of GFS (Graphical File System), MagReduce and BgTable, which are the core technologies of Google cloud computing. Hadoop's ecological map is shown in Figure 4.

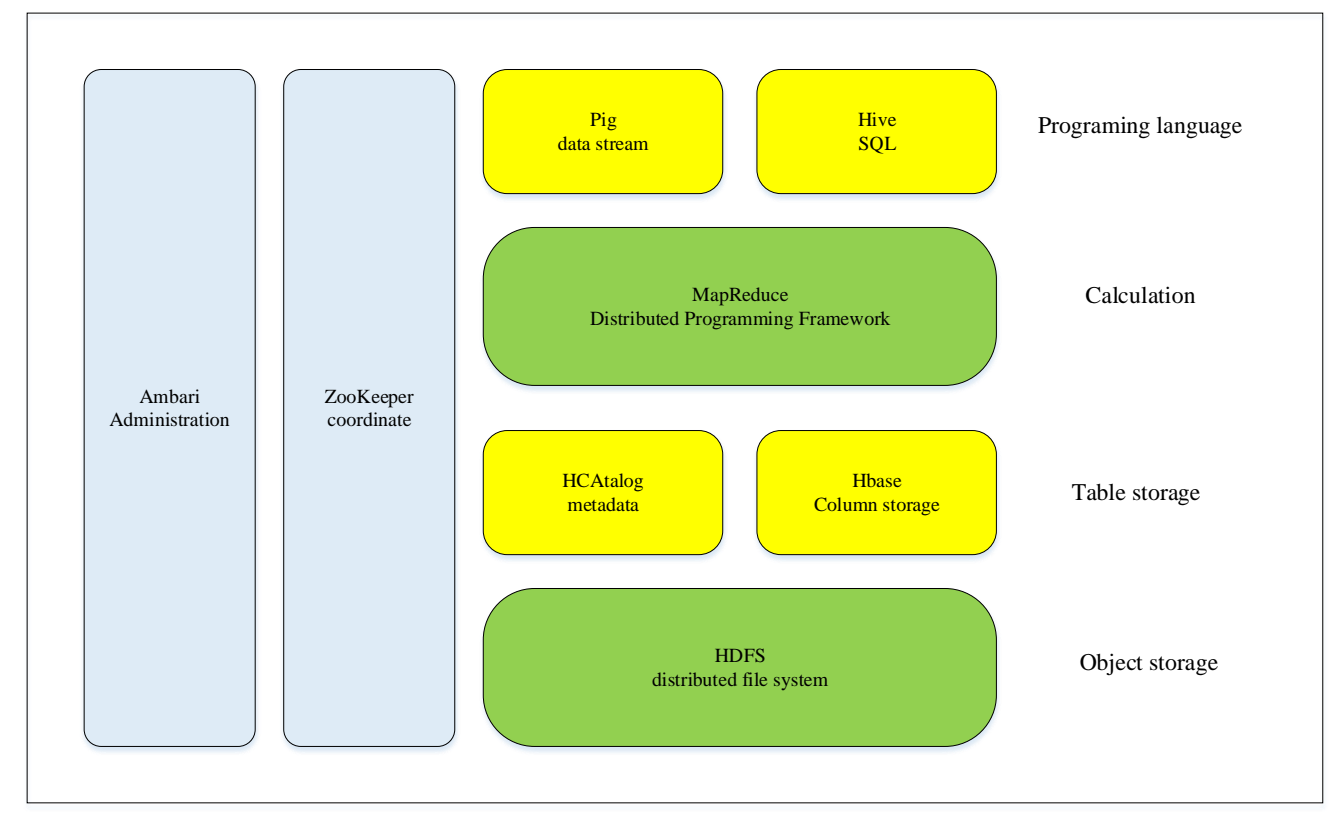

Figure 4: Hadoop ecological map 
Cloud computing, as a new network computing mode, is the result of the mixed evolution of a variety of technologies and driven by major giants, which has developed very rapidly in recent years.

At present, Google, IBM (International Business Machine), Amazon, Microsoft and other companies have launched their own cloud computing services, such as mail, search, network storage, document processing, parallel computing and a large number of Web applications.

Typical cloud computing platforms include Microsoft's Windows Azure Platform, Google's cloud computing platform, IBM and Xen cloud platforms, etc.

\section{Design of Mechanical Fault Diagnosis System Based on Cloud Computing Technology}

\subsection{System requirement analysis}

Mechanical fault diagnosis technology is a professional technology which integrates knowledge of many fields. It plays an important role in ensuring the safety, stability, long period and full load operation of machinery.

In recent years, it is developing at an unimaginable speed. However, with the development of modern science and technology, all kinds of mechanical equipment tend to be large-scale, highspeed, high-efficiency, energy-saving and complex, which puts forward higher requirements for the safe operation of machinery.

At the same time, in order to solve the problems of real-time failure symptoms of units in production site and the analysis and processing of a large amount of information, it poses greater challenges to the mechanical equipment diagnosis engineering. With the rapid development of network technology, especially with the popularization based on cloud computing and the development of cloud computing, the construction of mechanical fault diagnosis system based on cloud computing technology has incomparable advantages.
Specifically, it has the following characteristics: real-time, sharing, professionalism, economy and reliability [13].

The design of the system mainly considers the following aspects: following certain design principles, such as openness, expansibility, portability, and practicability; meeting the safety production requirements of machinery, continuously improving and enhancing the level of equipment maintenance and management; improving the response speed of the system and reducing the redundancy of the system's storage data and operation process.

The development of human-computer friendly interactive interface enables operators to easily grasp the operating rules of the system, and has high security.

The mechanical condition monitoring and fault diagnosis system based on cloud computing technology runs in a public network environment.

First, effective measures are taken to ensure the security of network transmission and prevent the leakage of enterprise information.

A prominent feature of mechanical fault diagnosis system based on cloud computing technology is that it can clearly divide the roles involved in the development of the system, so that each functional module can be continuously updated and improved according to actual needs.

The design of the system adopts the following design principles: low price and high performance.

\subsection{System design}

The mechanical condition monitoring and fault diagnosis system based on cloud computing technology adopts Browser/Server mode, which is Web server-centric, TCP/IP technology, HTTP as transmission protocol, and client can access the Web server and the background database connected with it through browser.

The system consists of three layers of architecture: client, system management, and infrastructure, as shown in Figure 5. 


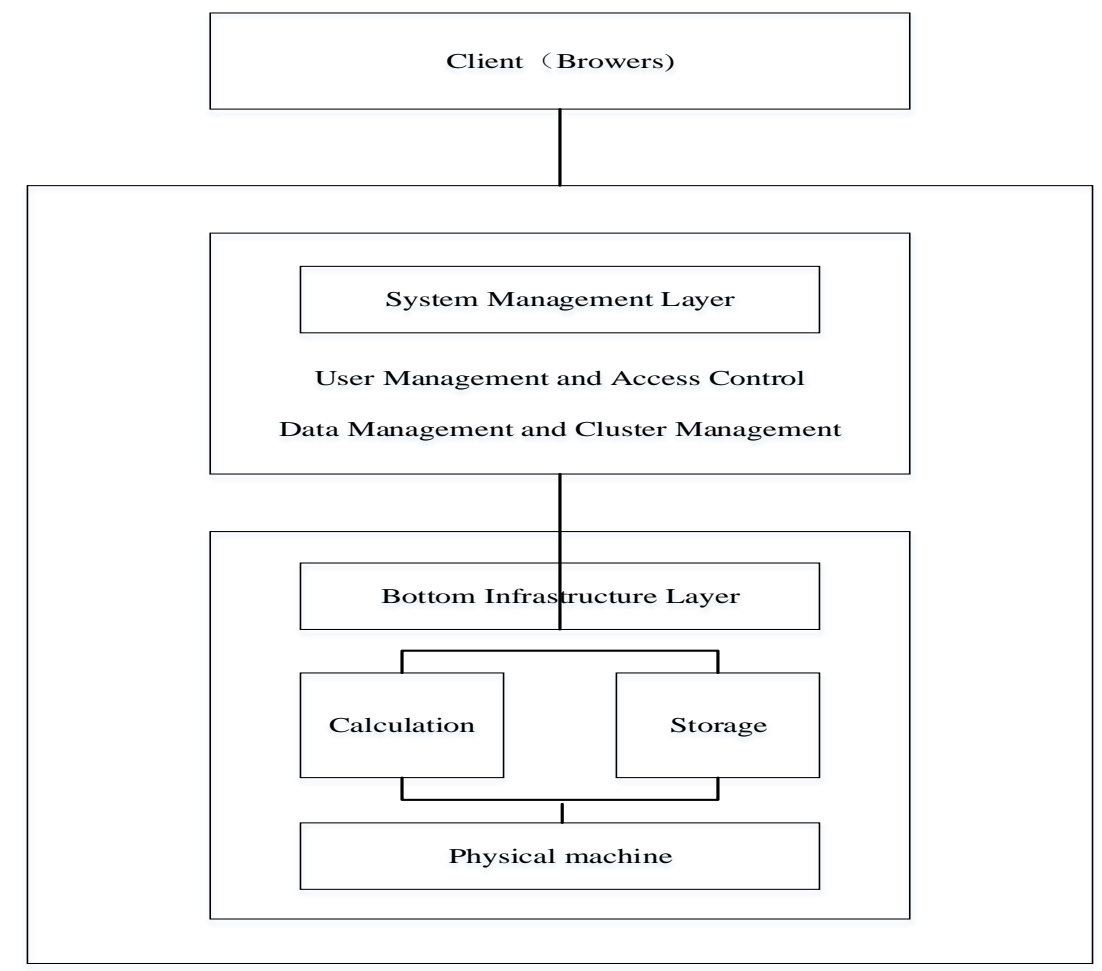

Figure 5: System architecture

The mechanical condition monitoring and fault diagnosis system based on cloud computing technology is a service platform of the system. The platform can expand hardware and software facilities by using distributed storage, parallel computing, virtualization and other technologies, effectively combine different resources and work together.

According to the functional requirement analysis of mechanical condition monitoring and fault diagnosis, the system is divided into four functional modules, including user login module, cluster monitoring module, file management module, and signal analysis and fault diagnosis module. Figure 6 is the framework of the functional modules of the system.

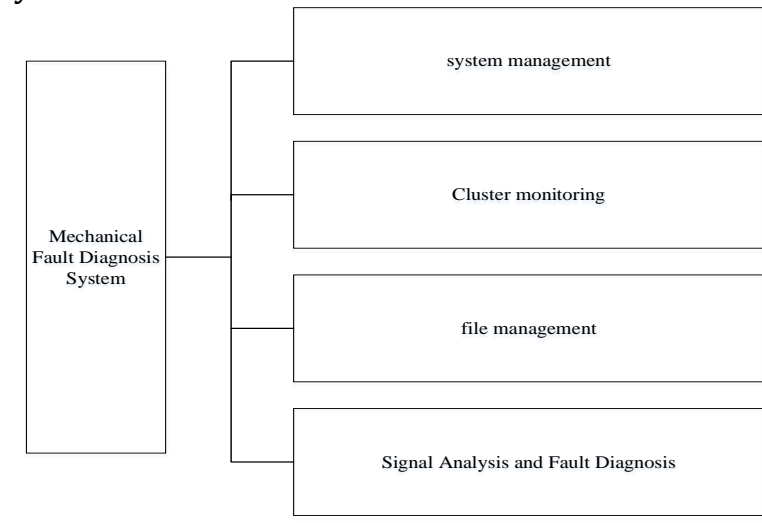

Figure 6: Block diagram of system function module

The system management module mainly includes two functions: access control and privilege management. User login, query, add, modify and delete, only the system administrator can achieve these operations. The user login system process is shown in Figure 7.

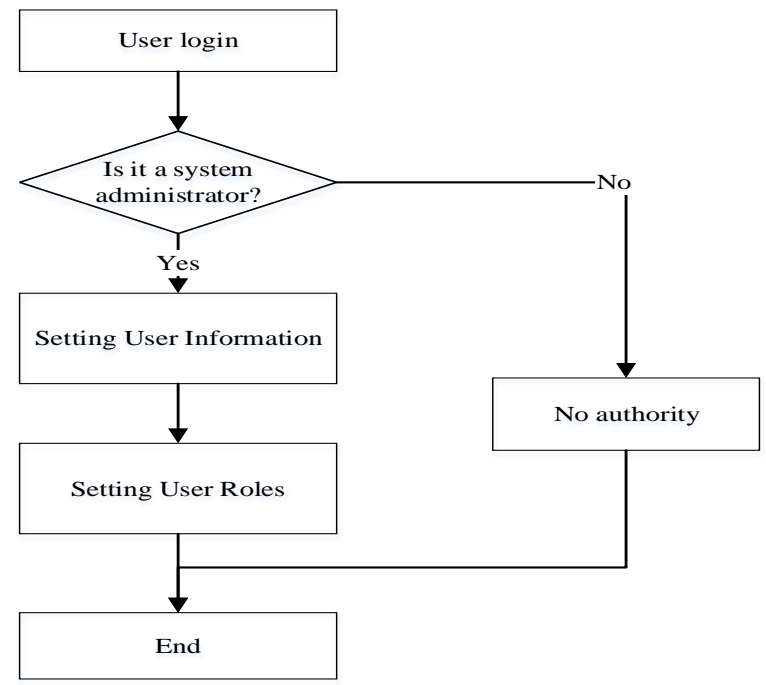

Figure 7: Flow chart of user login system

In addition to conventional analysis functions, such as time domain waveform, spectrum, axis trajectory, phase spectrum, cepstrum, polar coordinate map, signal analysis and diagnosis module can also add some modern signal analysis methods, like holographic spectrum analysis, vector spectrum analysis, wavelet analysis, Winger distribution and so on.

The function realization of signal analysis and fault diagnosis is mainly composed of four files, as shown in Table 2. 


\section{Table. 2 Functional module}

\begin{tabular}{|l|l|}
\hline $\begin{array}{l}\text { Program } \\
\text { name }\end{array}$ & Function \\
\hline cloud.jsp & $\begin{array}{l}\text { Interface of machinery on-line } \\
\text { monitoring and fault diagnosis } \\
\text { system. }\end{array}$ \\
\hline $\begin{array}{l}\text { datatransfer } \\
\text { java }\end{array}$ & $\begin{array}{l}\text { The data received by the server } \\
\text { is transmitted to the main } \\
\text { server, and the calculation } \\
\text { results are transmitted to the } \\
\text { client in the form of XML files. }\end{array}$ \\
\hline server.c & $\begin{array}{l}\text { It receives Java server-side data } \\
\text { and calls fft.c program. }\end{array}$ \\
\hline fft.c & $\begin{array}{l}\text { It is a parallel Fourier change } \\
\text { program. }\end{array}$ \\
\hline
\end{tabular}

In the field diagnosis of industrial production, spectrum analysis method is a very simple and effective fault analysis method. The purpose of spectrum analysis is to separate the various components of the signal. For rotating machinery and equipment, the frequency component of vibration signal has a great correlation with rotating speed, usually the integral or fractional times of rotating frequency [14].

The parallel program flow is shown in Figure 8.

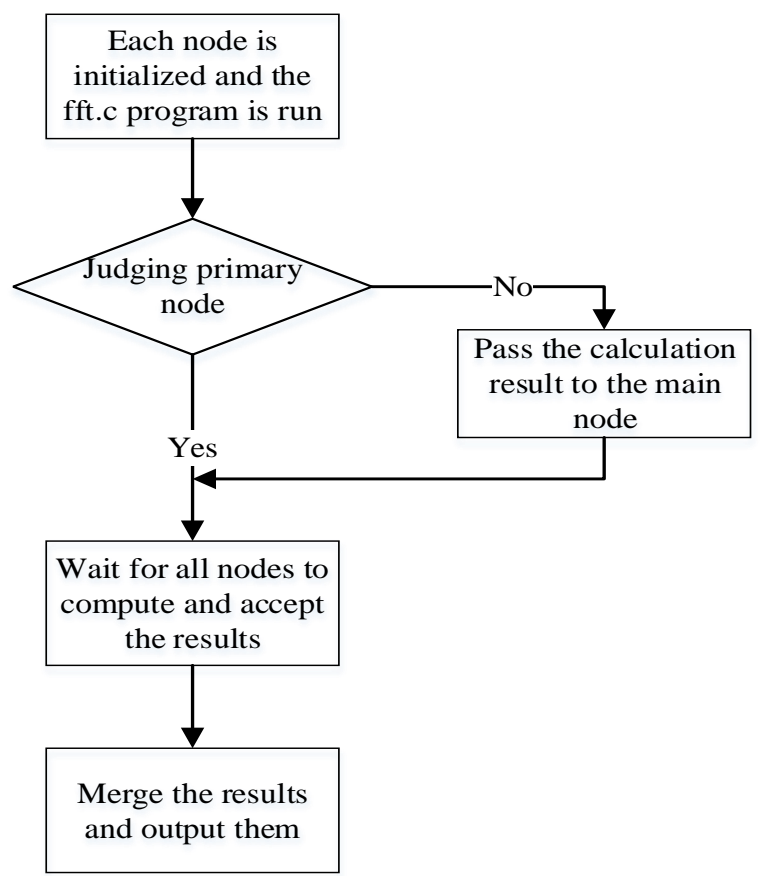

Figure 8: Parallel FFT flow chart

\subsection{Database design}

Apache server software is used as service application platform, Java language is used to design pages, and Mysql is used to design database. Apache server software's web page file directory defaults to be in the file path / var / www. It is only necessary to put the program code written in Java language into it to access the page through the browser.

MYSQL CLUSTER database cluster technology is used to construct cluster database. MYSQL CLUSTER is a database cluster technology. This technology allows the deployment of database Cluster in nonshared systems. The system usually uses cheap hardware to deploy the database. Cluster is composed of three different nodes: management node (ndb_mgmd), data node (Data Nodes), and SQL node (mysqld), which constitute a complete MYSQL cluster system.

Four computers are installed and configured database, mysql-server is installed, MySQL configuration file is edited, and MySQL configuration file of host node is configured. Then, the database connection method is abstracted into a class file.

According to the function and process design of the system, different data tables need to be designed, which are divided into four categories: user login related data tables, cluster node related data tables, file storage related data tables, and mechanical failure information related data tables.

The login management module needs to design three data tables, namely user information table (uin for_table), service information table (serve_table), and order_table. Set up another system monitoring data table to record. File management includes data storage structure and file index. It uses three tables: memory_table, file_table and block_table.

\subsection{MapReduce implementation of parallel FFT algorithms}

The implementation process of FFT parallel algorithm is as follows:

Discrete Fourier transform:

$$
y_{k}=\sum_{n=0}^{N-1} x_{n} \times W_{N}^{n k}
$$

$W_{n}=\exp (-j 2 \pi / N) ; \mathrm{k}=0,1, \ldots, \mathrm{N}-1$ is written into matrix form:

$$
\begin{aligned}
& \overrightarrow{Y^{T}}=B_{N} \overrightarrow{X^{T}} \\
& B_{N}=\left[\begin{array}{l}
W_{N}^{0}, W_{N}^{0} \cdots W_{N}^{0} \cdots W_{N}^{0} \\
W_{N}^{0}, W_{N}^{1} \cdots W_{N}^{n} \cdots W_{N}^{N-1} \\
\cdots, \cdots, \cdots, \cdots, \cdots, \cdots \\
W_{N}^{0}, W_{N}^{k} \cdots W_{N}^{n k} \cdots W_{N}^{n} \\
\cdots, \cdots, \cdots, \cdots, \cdots, \cdots \\
W_{N}^{0}, W_{N}^{N-1} \cdots W_{N}^{n(N-1)} \cdots
\end{array}\right. \\
& \overrightarrow{X_{T}}=\left(x_{0}, x_{1}, \cdots x_{N-1}\right)^{T} \\
& \overrightarrow{Y_{T}}=\left(y_{0}, y_{1}, \cdots y_{N-1}\right)^{T}
\end{aligned}
$$$$
B_{N}=\left[\begin{array}{l}
W_{N}^{0}, W_{N}^{0} \cdots W_{N}^{0} \cdots W_{N}^{0} \\
W_{N}^{0}, W_{N}^{1} \cdots W_{N}^{n} \cdots W_{N}^{N-1} \\
\cdots, \cdots, \cdots, \cdots, \cdots, \cdots \\
W_{N}^{0}, W_{N}^{k} \cdots W_{N}^{n k} \cdots W_{N}^{n(\mathrm{~N}-1)} \\
\cdots, \cdots, \cdots, \cdots, \cdots, \cdots \\
W_{N}^{0}, W_{N}^{N-1} \cdots W_{N}^{n(N-1)} \cdots W_{N}^{(\mathrm{N}-1)(\mathrm{N}-1)}
\end{array}\right]
$$

From the above formula, it can be seen that FFT is easy to realize parallel computing and has the characteristics of network parallel computing. 
Parallel FFT algorithm is implemented by Hadoop's MapReduce model. The algorithm flow is designed as follows: First, under the MapReduce parallel programming model, the computing load is allocated for each mapping task, and different key information is configured. Finally, mapping tasks are generated in sequence $\left(x_{0}, x_{1}, \cdots, x_{n-1}\right)$. The mapping task is assigned to each computing node according to different key information, and the Map function is called to perform the block operation task. The sequence is divided into two groups: even item sequence $\left(a_{0}, a_{1}, \cdots, a_{n-1}\right)$ and odd item sequence $\left(b_{0}, b_{1}, \cdots, b_{n-1}\right)$, and the calculation results are stored in different arrays according to load balancing. The Reduce function is called to perform the protocol operation. When each cluster computing node performs the Reduce task, the values in the same key are merged and output.

Assuming that the time for the processor performing one complex addition operation is $t_{1}$ and that for one complex multiplication operation is $t_{2}$, the time required for a single processor to complete $\mathrm{N}$-point FFT is:

$$
\begin{aligned}
& T_{1}=\frac{N}{2} \log _{2} N \cdot t_{2}+N \log _{2} N \cdot t_{1} \\
& =N \log _{2} N\left(\frac{t_{2}}{2}+t_{1}\right) \approx 21 N \log _{2} N \cdot t_{1}
\end{aligned}
$$

The time required to implement N-point FFT in parallel computing of $\mathrm{M}$ nodes, the acceleration ratio $S_{m}$ and the efficiency $E_{m}$ of parallel computing are shown below, respectively:

$$
\begin{aligned}
& T_{2}=\frac{1}{2^{m}}\left(\frac{N}{2} \log _{2} N \cdot t_{2}+N \log _{2} N \cdot t_{1}+m N \cdot 3 t_{1}\right) \\
& \approx \frac{N}{2^{m}}\left(21 \log _{2} N+3 m\right) \cdot t_{1} \\
& S_{m}=\frac{T_{1}}{T_{2}}=\frac{21 \log _{2} N \cdot t_{1}}{\frac{N}{2^{m}}\left(21 \log _{2} N+3 m\right) t_{1}} \\
& =\frac{7 \log _{2} N}{7 \log _{2} N+m} \\
& E_{m}=\frac{S_{m}}{m}
\end{aligned}
$$

Generally, the parallel FFT algorithm of cluster nodes takes routing time into account, but when designing parallel FFT algorithm under Hadoop framework, this structure just satisfies the direct routing and does not need to consider the problem of routing time.

\section{System Implementation}

\subsection{Construction of Hadoop cluster development environment}

A cloud platform for experiment is built on four hosts. Besides belonging to the same network segment, the username and password of the four hosts should be the same (easy to connect and login). Taking Hadoop user name as an example, installation configuration and experimental analysis all use this user name for related operations. In the self-built cloud computing environment in the laboratory, the machine names and corresponding IP addresses of each host are shown in Table 3.

Table 3. Machine name and IP address of each host

\begin{tabular}{|l|l|l|}
\hline Function & $\begin{array}{l}\text { Machine } \\
\text { name }\end{array}$ & IP address \\
\hline $\begin{array}{l}\text { Master node } \\
\text { (Namenode) }\end{array}$ & $\begin{array}{l}\text { Hadoop. } \\
\text { master }\end{array}$ & 192.168 .1 .239 \\
\hline $\begin{array}{l}\text { Slave node } \\
\text { (Datanode) }\end{array}$ & $\begin{array}{l}\text { Hadoop. } \\
\text { slave91 }\end{array}$ & 192.168 .1 .238 \\
\hline $\begin{array}{l}\text { Slave node } \\
\text { (Datanode) }\end{array}$ & $\begin{array}{l}\text { Hadoop. } \\
\text { slave92 }\end{array}$ & 192.168 .1 .237 \\
\hline $\begin{array}{l}\text { Slave node } \\
\text { (Datanode) }\end{array}$ & $\begin{array}{l}\text { Hadoop. } \\
\text { slave93 }\end{array}$ & 192.168 .1 .236 \\
\hline
\end{tabular}

Software configuration: Red Hat Linux operating system and sun-java6-jdk package need to be installed on each machine. Cloud computing development platform Hadoop0.20 is also installed in cluster environment. Tomcat6.0 is used in WEB server, which is the running platform for program development, compilation, and debugging. MYSQL6.0 database is used and each machine is also equipped with OPENSSH server to realize password-free access between clusters.

Hadoop is written in Java language, so Java JDK needs to be installed first. JDK is the basic development environment of Hadoop framework, distributed storage, and cloud computing platform.

\subsection{System function realization}

Users first enter the system logging page. Cloud computing cluster monitoring management module mainly monitors the working status of cluster nodes, including data monitoring, threshold setting, mechanical parameters acquisition, and node control. Cluster node status information, such as CPU utilization, memory utilization, network card flow, and surviving node information and storage capacity can be viewed, and the use of the file system can be browsed. By setting the threshold, it can accurately judge whether the state before the node is normal or not, and decide whether to add the node according to the traffic demand. 
File storage module mainly realizes distributed storage, parallel upload and download of files. The master node of the server cluster firstly classifies the uploaded files according to the key information, receives the number of data blocks from the client by calling the getnodeaddrthread () function, and allocates a storage node for each data block. When downloading files, first query the basic information of metadata in HDFS, and establish a data connection, which downloads data blocks from each storage node in parallel to the local, and then integrates them into a complete data file.

The experimental data used in mechanical signal analysis and fault diagnosis module are composed of motor, torque sensor/decoder, dynamometer, and electrical control device. Using the acceleration sensor, the two sensors are installed on the bearing seat near the motor drive end in 90 degree direction. The rotate speed of the rotor is $1979 \mathrm{rpm}$. 512 points of fault data (the data unit is voltage: $U / V$ ) of the outer ring of the bearing at the drive end are obtained by sampling frequency of $f_{s}=12000 \mathrm{~Hz}$.

The known bearing type is 6205-2RS JEM SKF, the passing frequency of bearing outer ring fault is $106.2 \mathrm{~Hz}$, and the failure frequency of bearing inner ring is $160 \mathrm{~Hz}$. The failure data are analyzed. It can be preliminarily judged that the rolling surface of the bearing has large local faults, which cannot meet the performance requirements of mechanical safety production. As for the fundamental causes and solutions of bearing failure, further analysis is needed.

\subsection{Analysis of experimental results}

The nodes used in this experiment use the same configuration, each node name is master, Node01, Node02, Node03, and master, as NameNode and JobTracer, also participates in the calculation.

The experimental results are shown in Table 4. S $(\mathrm{N})$ and $\mathrm{E}(\mathrm{N})$ represent the acceleration ratio and parallel efficiency of cluster nodes, respectively.

Table 4. Parallel FFT computing effect analysis table

\begin{tabular}{|l|c|c|c|c|c|}
\hline $\mathrm{N}$ & 256 & 512 & 1024 & 2048 & 4096 \\
\hline$T_{1} / \mathrm{ms}$ & 2.31 & 2.95 & 3.20 & 5.60 & 10.41 \\
\hline$t_{2} / \mathrm{ms}$ & 2.10 & 2.82 & 2.90 & 5.40 & 9.52 \\
\hline$t_{3} / \mathrm{ms}$ & 1.92 & 2.70 & 2.62 & 5.12 & 8.12 \\
\hline$S_{2}(\mathrm{~N})$ & 1.49 & 1.52 & 1.54 & 1.62 & 1.68 \\
\hline $\begin{array}{l}S_{3}(\mathrm{~N}) \\
E_{2}(\mathrm{~N}) / \\
\%\end{array}$ & 1.60 & 1.73 & 1.96 & 2.20 & 2.36 \\
\hline $\begin{array}{l}E_{3}(\mathrm{~N}) / \\
\%\end{array}$ & 36 & 39 & 40 & 43 & 53 \\
\hline
\end{tabular}

The algorithm experiment and analysis show that the more cluster nodes are, the more efficient parallel computing will be. MapReduce programming model is more suitable for solving data-intensive computing problems. Users only need to assign the computing unit tasks that a single node needs to complete, thus solving the complex programming problems in parallel computing.

A mechanical fault diagnosis system based on cloud computing is designed and developed. An open source Hadoop framework is used to build the computing development environment. The parallel FFT algorithm is programmed using MapReduce parallel programming model. The parallel computing and distributed storage of cluster data in low-end computers are realized through the deployment and testing of the program.

\section{Conclusion}

Since cloud computing was proposed, its distributed parallel computing and high data reliability have attracted wide attention. Among them, Hadoop, a highly scalable platform, can store and distribute data to hundreds of low-cost parallel operation server clusters. Deploying data to cloud platform for rapid filtering and efficient analysis has become the main way of data processing in the information society. Combining cloud computing technology with mechanical fault diagnosis technology, the construction scheme implementing mechanical fault diagnosis in cloud computing environment is proposed, and the following work is completed.

The theory and development status of mechanical fault diagnosis technology at the present stage, as well as the characteristics of various fault types and their analysis methods are introduced and analysed; cloud computing theory, development status, key technologies and typical cloud computing platforms are briefly introduced, and the Hadoop framework of open source cloud computing platform is analysed; the implementation methods of mechanical fault diagnosis system based on cloud computing technology are analysed, such as distributed storage of data, programming of parallel FFT algorithm and so on. It provides a new idea for the application and development of cloud computing technology, and successfully builds Hadoop in cloud computing environment and database cluster composed of lowend computers. Based on MapReduce programming model, the parallel algorithm is programmed, and the execution process and performance of the program are analysed theoretically. The functional modules of the system, including login module, cluster node monitoring module, data storage module and signal analysis and fault diagnosis module, are developed on Hadoop platform combined with WEB technology. 
Although cloud computing technology has been widely used and developed in various industries, the application of cloud computing technology in various fields is still in an initial stage, some key technologies are not mature, and the system performance needs to be further improved. The mechanical fault diagnosis system based on cloud computing is a simple application of cloud computing technology.

With the maturity and perfection of cloud computing technology, the development of mechanical fault diagnosis system will be the very complex and professional system engineering. The mechanical fault diagnosis system designed and developed initially realizes the distributed storage and parallel Fourier transform of data in cloud computing environment, and completes the deployment and testing of the program. However, the system functions need to be further improved, and some key technologies need further research and breakthroughs.

There are still some problems to be improved, including: the efficiency of MapReduce implementation is closely related to Hadoop parameter configuration, and a lot of experiments are needed to obtain the optimal configuration parameters and further obtain the configuration rules. To improve the parallelization and processing efficiency of FFT algorithm, it is necessary to continue to improve the FFT algorithm under MapReduce model. The visual interface of the system needs to be improved in order to meet the needs of users, that is, to refine and enrich functional modules so that operators can use them more conveniently and intuitively. Cloud computing platform is used to capture, analyse and process the field data in real time to realize intelligent operation, and combine with Internet of Things technology to truly realize the information production of equipment manufacturing industry.

In addition, due to the uncertainty of the underlying facilities of cloud computing and the networking of applications, the data stored in the cloud can easily be lost or tampered with, and even lead to system collapse, which will bring huge disaster to enterprises.

Therefore, the security of cloud computing needs to be further studied.

\section{Acknowledgement}

Research startup subject of Yangtze Normal Universi ty: 2017KYQD16

\section{References}

[1] Kumar, A., Shankar, R., Choudhary, A., \& Thakur, L. S. (2016). A big data mapreduce framework for fault diagnosis in cloud-based manufacturing. International Journal of Production Research, 54(23), 14.

[2] Yuan, C., Peng, L., \& Yuzhuo, Z. (2018). Parallel processing algorithm for railway signal fault diagnosis data based on cloud computing. Future Generation Computer Systems, 88, 279283.

[3] Zhang, C. H., Cheng, S. J., \& Cao, S. H. (2014). The support vector machine fault diagnosis algorithm based on cloud computing. Applied Mechanics and Materials, 687-691, 5.

[4] Wan, L., Han, G., Shu, L., Feng, N., Zhu, C., \& Lloret, J. (2015). Distributed parameter estimation for mobile wireless sensor network based on cloud computing in battlefield surveillance system. IEEE Access, 3, 1729-1739.

[5] Zhou, Q., Sun, C., Liao, R., Li, J., Zhang, Y., \& Wang, S. (2014). Multiple fault diagnosis and short-term forecast of transformer based on cloud theory. High Voltage Engineering, 40(5), 1453-1460.

[6] Zhang, H. (2014). Manufacturing information based on cloud service platform. Advanced Materials Research, 971-973, 5.

[7] Zhu, C., Sheng, Z., Leung, V. C. M., Shu, L., \& Yang, L. T. (2015). Toward offering more useful data reliably to mobile cloud from wireless sensor network. IEEE Transactions on Emerging Topics in Computing, 3(1), 84-94.

[8] Abbas, A., Zhang, L., \& Khan, S. U. (2015). A survey on context-aware recommender systems based on computational intelligence techniques. Computing, 97(7), 667-690.

[9] Suneja, S., Isci, C., Bala, V., De Lara, E., \& Mummert, T. (2014). Non-intrusive, out-ofband and out-of-the-box systems monitoring in the cloud. ACM SIGMETRICS Performance Evaluation Review, 42(1), 249-261.

[10] Parekh, M., \& Saleena, B. (2015). Designing a cloud based framework for healthcare system and applying clustering techniques for region wise diagnosis. Procedia Computer Science, 50, 537-542.

[11] Masegosa, A. R., Martinez, A. M., \& Borchani, H. (2016). Probabilistic graphical models on multicore cpus using java 8. IEEE Computational Intelligence Magazine, 11(2), 41-54.

[12] Morgan, J., \& Garret E. O’Donnell. (2015). Cyber physical process monitoring systems. Journal of Intelligent Manufacturing, (5-8), 1-12.

[13] Friedrich, C., Lechler, A., \& Verl, A. (2014). Autonomous systems for maintenance tasks requirements and design of a control architecture. Procedia Technology, 15, 596-605.

[14] Benazzouz, Y., Aktouf, O. E. K., \& Parissis, I. (2014). A fault fuzzy-ontology for large scale fault-tolerant wireless sensor networks. Procedia Computer Science, 35, 203-212. 